\title{
Intersubband Plasmon Polaritons in Multiple Quantum Well Structures
}

\author{
W. ZIĘTKOWSKI* AND M. ZAŁUŻNY \\ Institute of Physics, M. Curie Skłodowska University \\ Pl. M. Curie Skłodowskiej 1, 20-031 Lublin, Poland
}

\begin{abstract}
Radiative intersubband plasmon polaritons in multiple quantum well structures, with arbitrary number of quantum wells, are discussed theoretically. The dispersion relations are derived modeling multiple quantum well structure by uniform uniaxial effective medium and using a full set of the Maxwell equations for macroscopic (local) electromagnetic fields. Numerical calculations are performed for a "GaAs-GaAs/AlGaAs multiple quantum well-air" structure corresponding to a typical total internal reflection geometry. Manifestation of these modes in total internal reflection spectra is also considered.
\end{abstract}

PACS numbers: 78.66.Fd, 73.20.Mf, 71.36.+c

Radiative (virtual) polaritons are electromagnetic modes of optically-active layered structures, which migrate outside the sample in the form of radiation. These modes are very useful in discussion of optical properties of multilayer systems. It is connected with the fact that, in contrast to nonradiative modes, they can couple with external electromagnetic waves. For the first time radiative modes were discussed by Kliewer and Fuchs in Ref. [1]. (These authors investigated excitations of the ionic crystal slab connected with phonon-photon interaction.) They showed that virtual polaritons are characterized by complex frequency $\omega=\omega^{\prime}+i \omega^{\prime \prime}$. Contribution to the absorption spectra due to an individual virtual mode is a resonance peak centered at $\omega^{\prime}$ with total width $2\left|\omega^{\prime \prime}\right|$. The imaginary part of the frequency is connected with the radiative and scattering damping.

In this paper we want to adopt virtual mode approach to describe optical properties of multiple quantum well in the frequency range corresponding to intersubband transitions. We will consider radiative polaritons which are a mixture of intersubband plasmons and photons. (Nonradiative intersubband plasmon polaritons will be a subject of forthcoming article.)

*corresponding author; e-mail: wzietko@tytan.umcs.lublin.pl 
Properties of the intersubband transitions (ITs) are discussed in detail in the review article by Helm [2]. Bulk intersubband plasmon polaritons (IPPs) in infinite superlattices are investigated by King-Smith and Inkson [3] in the fully nonlocal approach. The radiative and nonradiative IPPs in a single quantum well (SQW) structure are discussed by Chen et al. [4] in the framework of the so-called local slab model and Wendler et al. [5,6] (see also Ref. [7]) employing the fully nonlocal approach.

Below we discuss dispersion properties of the radiative intersubband plasmon polaritons (RIPPs) in a typical "dielectric-MQW-air" systems which are commonly used in total internal reflection (TIR) setup experiments. The dispersion relations of radiative modes will be derived modeling an MQW structure (with arbitrary number of quantum well $N_{\mathrm{QW}}$ ) by an effective uniaxial local medium $[8,9]$. The usefulness of the local susceptibility model for description of optical properties of MQW structures (connected with RIPPs) has been recently demonstrated by Dupont et al. [10].

As was mentioned, in this paper the MQW structure is treated as an effective uniform uniaxial medium. The diagonal dielectric tensor $\varepsilon(\omega)$ describing the optical properties of such a medium can be written in the following form [8]:

$$
\begin{aligned}
& \varepsilon_{j j}(\omega)=\epsilon_{x x}+\Delta \varepsilon_{x x}(\omega), \quad j=x, y, \\
& \varepsilon_{z z}^{-1}(\omega)=\epsilon_{z z}^{-1}-\Delta \varepsilon_{z z}(\omega) / \varepsilon_{\mathrm{W}}^{2}, \\
& \Delta \varepsilon_{x x}(\omega)=\mathrm{i} 4 \pi \sigma_{x x}^{2 \mathrm{D}}(\omega) / \omega L_{\mathrm{MQW}}, \\
& \Delta \varepsilon_{z z}(\omega)=\mathrm{i} 4 \pi \tilde{\sigma}_{z z}^{2 \mathrm{D}}(\omega) / \omega L_{\mathrm{MQW}},
\end{aligned}
$$

where $\epsilon_{x x}=(1-f) \varepsilon_{\mathrm{b}}+\mathrm{f} \varepsilon_{\mathrm{w}}, \epsilon_{z z}^{-1}=(1-f) / \varepsilon_{\mathrm{b}}+f / \varepsilon_{\mathrm{W}}, \varepsilon_{\mathrm{b}}\left(\varepsilon_{\mathrm{w}}\right)$ is the background dielectric constant of the barrier (well) material, $f=L_{\mathrm{QW}} / L_{\mathrm{MQW}}, L_{\mathrm{MQW}}$ is the period of the MQW structure and $L_{\mathrm{QW}}$ is the thickness of the $\mathrm{QW}$. (We assume for simplicity that the QWs have rectangular shape.)

The normal $2 \mathrm{D}$ conductivity $\tilde{\sigma}_{z z}^{2 \mathrm{D}}(\omega)$ describes (nonretarded) response of the quasi-two-dimensional electron gas (located in the single QW) to the $z$ component (normal to the growth direction) of the external electric field $\left[=\boldsymbol{E}(r) \mathrm{e}^{-\mathrm{i} \omega t}\right]$.

The expression for $\tilde{\sigma}_{z z}^{2 \mathrm{D}}(\omega)$ takes the form [11]:

$$
\tilde{\sigma}_{z z}^{2 \mathrm{D}}(\omega)=\frac{N_{\mathrm{s}} e^{2} f_{21}}{m} \frac{-\mathrm{i} \omega}{\tilde{\omega}_{21}^{2}-\omega^{2}-\mathrm{i} 2 \omega / \tau_{\perp}},
$$

where $\tilde{E}_{21} \equiv \hbar \tilde{\omega}_{21}$ is the intersubband transition energy modified by the depolarization and exciton-like effects, $f_{12}=2 m \hbar^{-2} E_{21} z_{21}^{2}$ is the oscillator strength connected with $1 \rightarrow 2$ transitions $\left(f_{12} \approx 1\right), \tau_{\perp}$ is the phenomenological dephasing time connected with $1 \rightarrow 2$ transitions, $N_{\mathrm{s}}$ is the surface electron concentration, and finally, $e$ and $m$ are the charge and effective mass of the electron, respectively. For simplicity we restrict ourselves to the two parabolic subband model (with the intersubband spacing $E_{21} \equiv \hbar \omega_{21}$ ) assuming that only ground subband is occupied. Since we work in the frequency range corresponding to intersubband 
transitions, the contribution of intrasubband transitions to $\varepsilon_{x x}$ is negligibly small. Due to this, in analytical calculations we take $\Delta \varepsilon_{x x}(\omega)=0$.

Using Eqs. (1) and (2), one finds that (in the dissipationless limit) IPPs in bulk effective medium obey the dispersion relation [12]:

$$
\omega_{ \pm}^{\mathrm{inf}}\left(k_{x}, k_{z}^{\mathrm{MOW}}\right)=\frac{1}{\sqrt{2}}\left[\left(\omega_{1}^{2}+\tilde{\omega}_{21}^{2}\right) \pm \sqrt{\left(\omega_{1}^{2}-\tilde{\omega}_{21}^{2}\right)^{2}+4 \Omega_{\text {inf }}^{4}}\right]^{1 / 2},
$$

where $\boldsymbol{k}=\left[k_{x}, 0, k_{z}^{\mathrm{MQW}}\right]$ is the polariton wave vector in the effective medium, $\omega_{1}^{2} \equiv \omega_{1}^{2}\left(k_{x}, k_{z}^{\mathrm{MQW}}\right)=c^{2}\left[k_{x}^{2} / \epsilon_{z z}+\left(k_{z}^{\mathrm{MQW}}\right)^{2} / \epsilon_{x x}\right], \Omega_{\text {inf }}^{4}=f_{12}\left(\omega_{p}^{\mathrm{MQW}} c k_{x}\right)^{2} \varepsilon_{\mathrm{W}} / \epsilon_{z z}$, and $\left(\omega_{p}^{\mathrm{MQW}}\right)^{2}=4 \pi N_{\mathrm{s}} e^{2} / m \varepsilon_{\mathrm{W}} L_{\mathrm{MQW}}$.

Let us consider TM eigenmodes that propagate in the $x-z$ plane in a three-phase system formed by MQW structure (medium 1) sandwiched between two semi-infinite materials. We assume that the region $|z|<d_{\mathrm{MQW}} / 2$ is occupied by MQW structure and the surrounding materials placed above (medium 0) and below (medium 2) the sample are characterized by dielectric constant $\varepsilon_{0}$ and $\varepsilon_{2}$ $\left(\varepsilon_{0}>\varepsilon_{2}\right)$, respectively. Since we work in the TIR regime, we assume that the angle of propagation $\varphi$ (in medium 0 ) is greater than the critical angle $\varphi_{c}^{\text {tir }}=$ $\arctan \left(\sqrt{\varepsilon_{2} / \varepsilon_{0}}\right)$. In another words, only these virtual modes which radiate from MQW structure to medium 0 (not medium 2), will be considered.

The components of electric field (in the $j$-th medium) connected with TM modes can be written in the following form:

$$
\begin{aligned}
& \begin{cases}E_{x}^{(0)}(z)=-B^{(0)} \mathrm{e}^{\mathrm{i} k_{z}^{(0)} z}, & z>d_{\mathrm{MQW}} / 2, \\
E_{x}^{(1)}(z)=A^{(1)} \mathrm{e}^{-\mathrm{i} k_{z}^{(1)} z}-B^{(1)} \mathrm{e}^{\mathrm{i} k_{z}^{(1)} z}, & |z|<d_{\mathrm{MQW}} / 2, \\
E_{x}^{(2)}(z)=A^{(2)} \mathrm{e}^{-\mathrm{i} k_{z}^{(2)} z}, & z<-d_{\mathrm{MQW}} / 2,\end{cases} \\
& \begin{cases}E_{z}^{(0)}(z)=\frac{k_{x}}{k_{z}^{(0)}} B^{(0)} \mathrm{e}^{\mathrm{i} k_{z}^{(0)} z}, & z>d_{\mathrm{MQW}} / 2, \\
E_{z}^{(1)}(z)=\frac{k_{x}}{k_{z}^{(1)}} \frac{\varepsilon_{x x}(\omega)}{\varepsilon_{z z}(\omega)}\left[A^{(1)} \mathrm{e}^{-\mathrm{i} k_{z}^{(1)} z}+B^{(1)} \mathrm{e}^{\mathrm{i} k_{z}^{(1)} z}\right], & |z|<d_{\mathrm{MQW}} / 2, \\
E_{z}^{(2)}(z)=\frac{k_{x}}{k_{z}^{(2)}} A^{(2)} \mathrm{e}^{-\mathrm{i} k_{z}^{(2)} z}, & z<-d_{\mathrm{MQW}} / 2,\end{cases}
\end{aligned}
$$

with

$$
k_{z}^{(j)}=\left\{\begin{array}{cl}
\sqrt{K^{2} \varepsilon_{j}-k_{x}^{2}}, & j=0,2, \\
\sqrt{\varepsilon_{x x}} \sqrt{K^{2}-k_{x}^{2} / \varepsilon_{z z}}, & j=1,
\end{array}\right.
$$

where $K=\omega / c$ is the wave vector of the radiation in vacuum.

Employing the standard boundary conditions for tangential components of electric field and normal components of displacement vector, we get [4]:

$$
\mathrm{i} \tan \left(k_{z}^{\mathrm{MQW}} d_{\mathrm{MQW}}\right)=\frac{\varepsilon_{x x}}{k_{z}^{\mathrm{MQW}}}\left(\frac{\varepsilon_{0}}{k_{z}^{(0)}}+\frac{\varepsilon_{2}}{k_{z}^{(2)}}\right)\left[\frac{\varepsilon_{0}}{k_{z}^{(0)}} \frac{\varepsilon_{2}}{k_{z}^{(2)}}+\left(\frac{\varepsilon_{x x}}{k_{z}^{\mathrm{MQW}}}\right)^{2}\right]^{-1},
$$

where $k_{z}^{\mathrm{MQW}}=k_{z}^{(1)}$. The electromagnetic (EM) excitations (polaritons) described by Eq. (10) can be generally divided into two classes: (i) nonradiative (bound) modes and (ii) radiative modes (for detail see Refs. [1, 4]). 


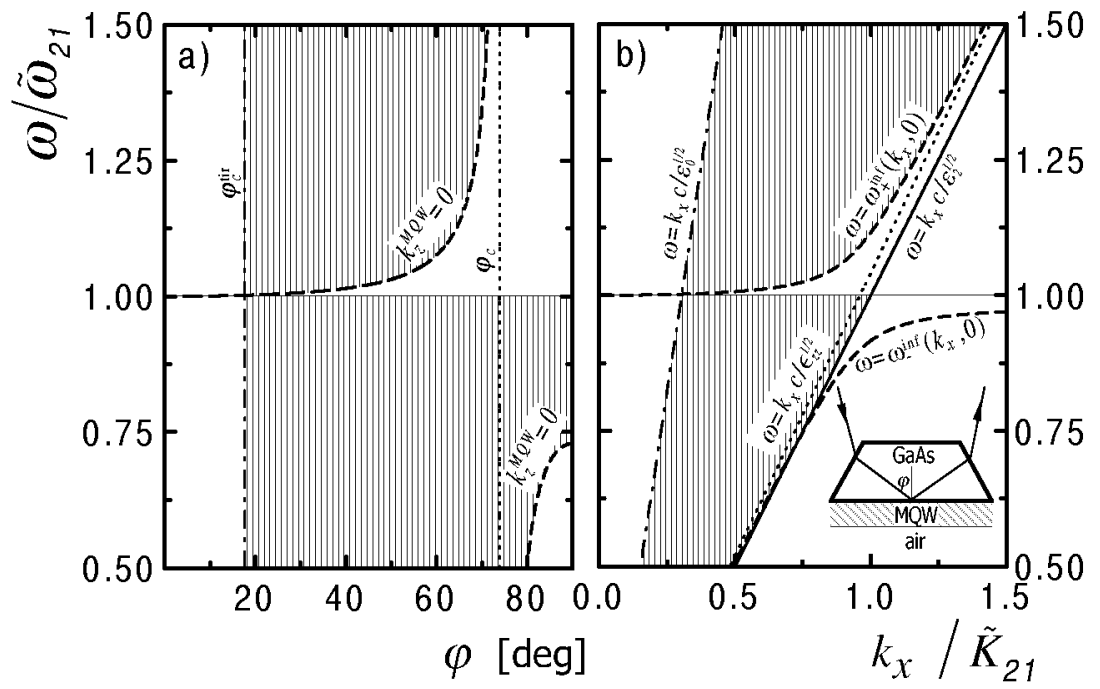

Fig. 1. Regions in the $\omega-\varphi$ plane (a) and the $k_{x}-\omega$ plane (b) for which RIPPs, considered in this paper, exist. $\tilde{K}_{21}=\sqrt{\varepsilon_{\mathrm{w}}}\left(\tilde{\omega}_{21} / c\right)$.
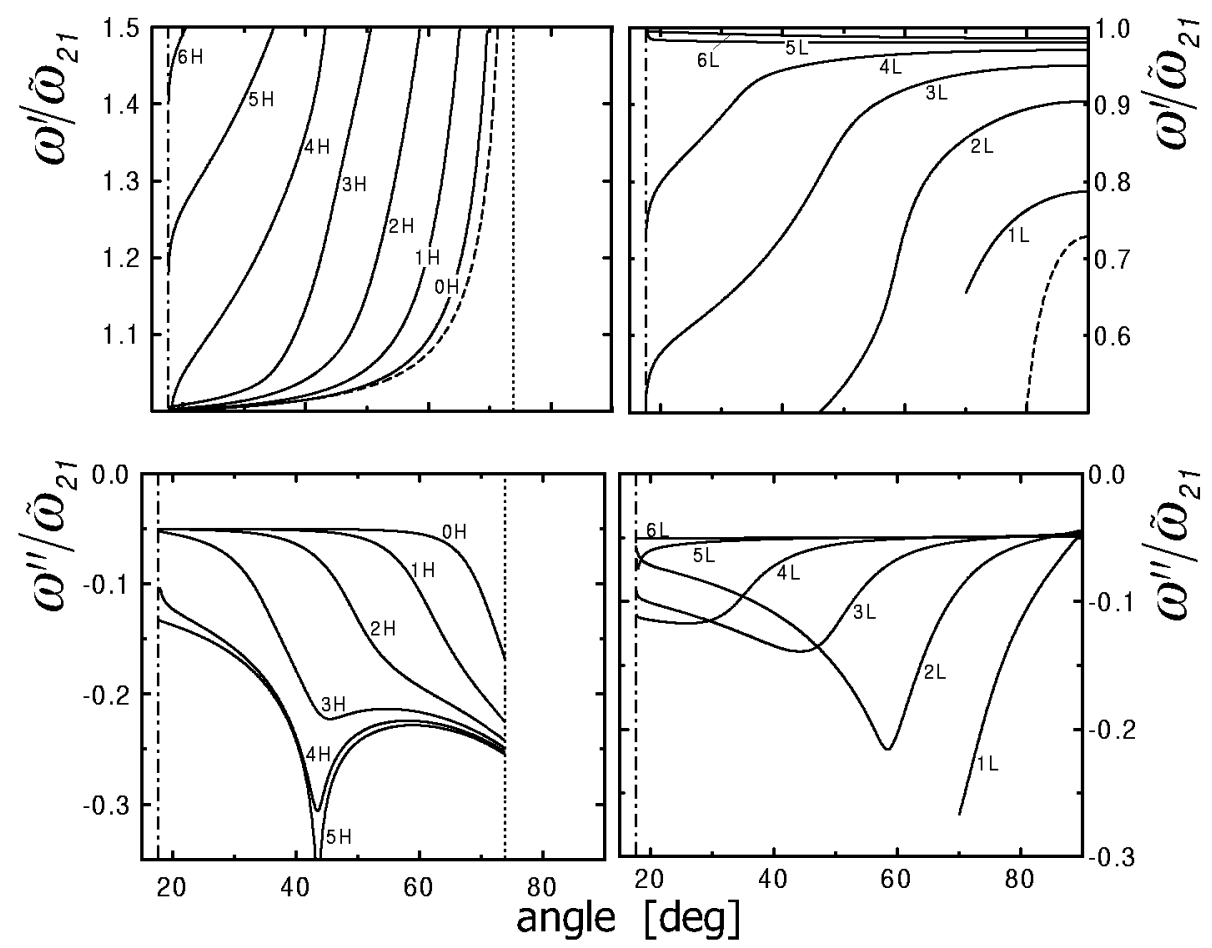

Fig. 2. Dispersion curves for high-frequency (left panels) and low-frequency (right panels) RIPPs in the "GaAs-MQW-air" system described in the main text. 
As mentioned in this paper, we consider only radiative modes radiating to the medium 0 , at fixed propagating angle $\varphi\left(>\varphi_{c}^{\text {tir }}\right)$. Wave vector, frequency and propagation angle of such modes should obey the following relations: $k_{x}=$ $\left|k_{x}\right| \mathrm{e}^{\mathrm{i} \alpha}, \omega=|\omega| \mathrm{e}^{\mathrm{i} \alpha}$ and $\varphi=\arctan \left|k_{x}\right| /\left|k_{z}^{(0)}\right|$, where $\alpha$ is real number [1].

Numerical calculations (see Fig. 1) are performed for a standard GaAs/ AlGaAs MQW structure similar to discussed in Ref. [8]: $\tilde{E}_{21}=111 \mathrm{meV}$, $f_{12}=0.85, N_{\mathrm{s}}=1.2 \times 10^{12} \mathrm{~cm}^{-2}, L_{\mathrm{MQW}}=405 \AA, \varepsilon_{\mathrm{W}}=10.9, \epsilon_{x x}=10.08$ and $\epsilon_{z z}=10.06$. Virtual modes can occur in the two (shaded) regions of $\varphi-\omega$ plane $\left(k_{x}-\omega\right.$ plane). Modes lying above $\tilde{\omega}_{21}$ are called high-frequency $(\mathrm{H})$ modes. Modes occurring below $\tilde{\omega}_{21}$ are called low-frequency $(\mathrm{L})$ modes.

Inspection of Eq. (10) indicates that a "GaAs-MQW-air" structure may support infinite number of radiative modes $(n=0,1,2 \ldots)$. Figure 2 presents dispersion curves for RIPPs in a GaAs-GaAs/AlGaAs MQW-air system with [8]: $\varepsilon_{0}=\varepsilon_{\mathrm{GaAs}}=10.9, \varepsilon_{2}=\varepsilon_{\mathrm{air}}=1\left(\varphi_{\mathrm{c}}^{\mathrm{tir}}=17.631^{\circ}\right), 2 \Gamma=2 \hbar \tau_{\perp}^{-1}=11.2 \mathrm{meV}$, $N_{\text {QW }}=200$. Figure 2 shows dispersion curves of high- and low-frequency modes, for $\omega$ close to $\tilde{\omega}_{21}$. It is worthy noting that high-frequency modes, in contrast to low-frequency modes, occur only for $\varphi$ smaller than critical angle $\varphi_{\mathrm{c}}=\arcsin \sqrt{\epsilon_{z z} / \varepsilon_{0}}$.

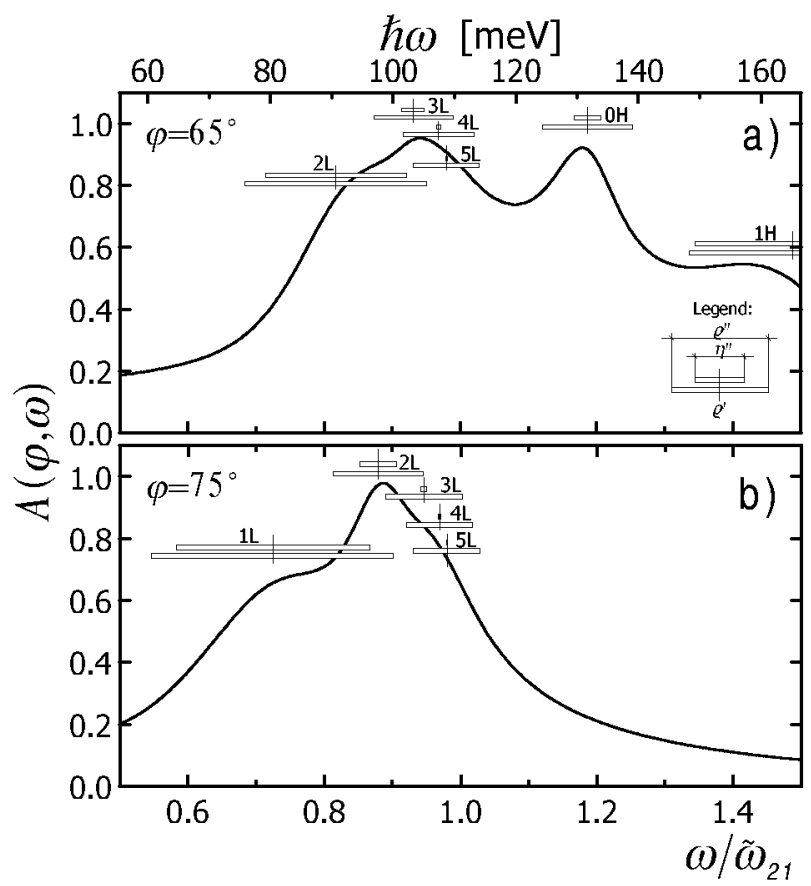

Fig. 3. Spectral dependence of $A(\varphi, \omega)$ calculated for the system discussed in Fig. 2, at $\varphi=65^{\circ}$ (a) and $\varphi=75^{\circ}$ (b). 
In the case of TIR geometry, in the absence of quasi-two-dimensional electron gas in MQW structure all the incident light will be reflected by the structure. Due to the intersubband excitations and damping, energy is removed from incident beam and the reflection coefficient $R(\omega, \varphi)$ is reduced to less than unity. As mentioned we would like to associate structure in absorption spectra "GaAs-MQW-air" system with RIPPs.

Figure 3 presents $\omega$-dependence of absorption $A(\omega, \varphi)=1-R(\omega, \varphi)$ calculated employing a transfer matrix method for $\varphi=65^{\circ}\left(<\varphi_{\mathrm{c}}\right)$ and $\varphi=75^{\circ}\left(<\varphi_{\mathrm{c}}\right)$. We are able to associate each peak (structure) with appropriate radiative mode. The double bar above each peak illustrates the center frequency $\rho^{\prime}$ of the virtual mode, its total width $2\left|\rho^{\prime \prime}\right|$ calculated with $\Gamma=11.2 \mathrm{meV}$, and its radiative width $2\left|\eta^{\prime \prime}\right|$ calculated with $\Gamma=0$. The results presented in Fig. 3 support usefulness of the virtual modes approach in the description of the infrared optical properties of MQW structures.

\section{References}

[1] K.L. Kliewer, R. Fuchs, Phys. Rev. B 144, 495 (1966); 150, 573 (1966); 150, $589(1966)$.

[2] M. Helm, in: Semiconductors and Semimetals, Vol. 62, Eds. H.C. Liu, F. Capasso, Academic Press, San Diego 2000, p. 1.

[3] R.D. King-Smith, J.C. Inkson, Phys. Rev. B 36, 4796 (1987).

[4] W.P. Chen, Y.J. Chen, E. Burstein, Surf. Sci. 58, 263 (1976).

[5] L. Wendler, E. Knadler, Phys. Status Solidi B 177, 9 (1993).

[6] L. Wendler, T. Kraft, Phys. Rev. B 60, 16603 (1999).

[7] A. Eguiluz, A.A. Maradudin, Ann. Phys. (USA) 113, 29 (1978).

[8] M. Załużny, C. Nalewajko, Phys. Rev. B 59, 13043 (1999).

[9] C. Nalewajko, Ph.D. thesis, M. Curie-Skłodowska University, Lublin 1999.

[10] E. Dupont, M. Gao, H.C. Liu, Z.R. Wasilewski, A. Shen, M. Załużny, S.R. Schmidt, A. Seilmeier, Phys. Rev. B 61, 13050 (2000).

[11] T. Ando, Z. Phys. B 24, 219 (1976).

[12] W. Ziętkowski, diploma thesis, M. Curie-Skłodowska University, Lublin 2000. 